\title{
Periodic One-Dimensional Hopping model with one Mobile Directional Impurity
}

\author{
Z. Toroczkai and R.K.P. Zia \\ Department of Physics and Center for Stochastic Processes \\ in Science and Engineering, Virginia Polytechnic Institute \\ and State University, Blacksburg, V.A. 24061-0435, USA
}

December 7, 2017

\begin{abstract}
Analytic solution is given in the steady state limit $t \rightarrow \infty$ for the system of Master equations describing a random walk on one-dimensional periodic lattices with arbitrary hopping rates containing one mobile, directional impurity (defect bond). Due to the defect, translational invariance is broken, even if all other rates are identical. The structure of Master equations lead naturally to the introduction of a new entity, associated with the walker-impurity pair which we call the quasi-walker. The velocities and diffusion constants for both the random walker and impurity are given, being simply related to that of the quasi-particle through physically meaningful equations. Applications in driven diffusive systems are shown, and connections with the Duke-Rubinstein reptation models for gel electrophoresis are discussed.
\end{abstract}

PACS: 02.50. $-r, 05.40 .+j, 05.60 .+w, 83.10 . N n$, 83.10.Pp

TO APPEAR IN J.STAT.PHYS 


\section{Introduction}

In equilibrium statistical mechanics the number of exactly (analyticaly) solvable models is very limited (for a review see [1] ). In nonequilibrium statistical mechanics this number is considerably smaller, and no review has been published, to our best knowledge, so far. Part of this difference lies in the starting points. In equilibrium physics, one usually begins with the well-known Boltzmann distribution. By contrast, arriving at this step is already non-trivial, even for steady states, since one must solve, say, a Master equation first. To find an analytical solution for such equations, for typical nonequilibrium systems, is usually impossible because of the very large number of different equations involved, since the unknown is a function of the configurations of the system. However, we can hope to find analytic solutions if the dimensionality of our system is low enough. As an example, consider the random walk of a single particle on a one dimensional (1D) lattice, with timeindependent hopping rates $W_{i, j}$, where $W_{i, j}$ represents the probability for the particle to jump from site $j$ to site $i$ in one step (or per unit time). These rates may be arbitrary, so that they are not required to satisfy the condition for detailed balance, so as to describe a general, nonequilibrium system. With the additional assumptions (a) that only jumps to the nearest sites are allowed $(|i-j|=1)$ and (b) that periodic boundary condition (PBC) is imposed, this model was analyticaly solved in the steady state limit by Derrida in 1983 (see [2]). Explicit expressions for the steady state distribution, velocity and diffusion constant were given. However, if, say, jumps to next nearest neighbours are allowed, an analytical solution seems to be impossible to give [2]. On the other hand, since 1983, several other types of 1D systems (e.g., [3], [4]) have been solved.

In the next two subsections of the Introduction we explicitely present on a "checkerboard" the one-dimensional periodic hopping model without and with a mobile directional impurity. Then we give a short description of the main body of the paper.

\subsection{One-dimensional hopping model without impurities}

The hopping model presented by Derrida in [2] can best be visualized as a $(N+1) \times 1$ checkerboard, i.e., a string of $N+1$ squares). This is filled with $N$ pieces (particles) numbered from 1 to $N$ (from left to right) and one hole, which will play the role of the random walker (Fig.1) . The periodic boundary condition is equivalent to identifying the squares at the two ends as nearest neighbours. By saying "that the hole is at site $n$ " we mean that the empty square is found in between particles with labels $n$ and $n+1$. Since the dynamics will be restricted to particle-hole exchange only, we may regard the hole as the random walker, and will use the terms'hole' and 'walker' interchangibly. The proposition "the hole jumps from site $n$ to site $n+1$ in the next time step" on Fig. 1 is equivalent to "shifting the hole from the square in between particles $n$ and $n+1$ to the square in between particles $n+1$ and $n+2$ ". The rules of evolution are as follows:

1) a square can be at most occupied by a single particle,

2) a particle can interchange squares only with the empty square (hole) and only if that is a nearest neighbour, 


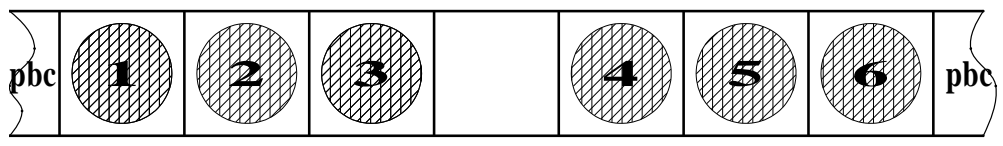

Figure 1: The translationally invariant one dimensional hopping model with periodic boundary conditions, on a checkerboard. The particles are represented by numbered circles (pieces).

3) the particles are not allowed to interchange positions among themselves, 4) whenever the hole at site $n$ jumps to the left (to site $n-1$ ), it does with probability $W_{n-1, n}$, and, for a jump to the right (to site $n+1$ ), the probability involved is $W_{n+1, n}$.

Note that the system above is translationally invariant, in the sense that the jump rate of any particular particle-hole pair is independent of its location on the checkerboard. In particular, after the hole jumps through the entire system a few times, (effectuating complete revolutions) the string of particles will be displaced, relative to the "board". Nevertheless, the physics of the evolution remains unaltered. Next, we break this invariance by introducing a "kink" in the checkerboard.

\subsection{The model with one mobile directional impurity}

In this paper we introduce a mobile, directional impurity into the periodic hopping model above. In terms of the checkerboard, this is a specific "bond" between two adjacent squares, such that rates of particle-hole exchanges across it are different (in the sense that they differ from the $W$ rates) . Thus, we also refer to it as a "defect bond" (DB). Since the string of particles can be displaced relative to the checkerboard, this DB can move, relative to the string. In this sense, we use the term "mobile directional impurity". A more transparent way to display the model is represented in Fig.2, where the DB is shown as a kink in the checkerboard. Only across this kink are the probabilities of the particle-hole exchange different from the $W$ 's specified above. The motivation for such a kink will be discussed below. Here, we simply state the model. In addition to the rules 1)-4) from the previous subsection, we have one more, specifying the jump rates through the kink:

5) the probabilities for the interchange of the hole with a particle through the DB (thick dashed line) are $q$, if the hole moves upward, and $q^{\prime}$, if downward, independent of the particle involved. This is a simplifying assumption, allowing less involved formulae. Our methods will lead to an analytic solution even if these probabilities do depend on the particle.

Note that, in both models, the order of the particles is never changed, i.e., there is no mixing among the particles. Therefore, when the hole is in between particles $n$ and $n+1$, the hopping probability to the left (right) is always $W_{n-1, n}\left(W_{n+1, n}\right)$, except when the DB is involved. However, the string of particles as a whole shifts with respect to the 


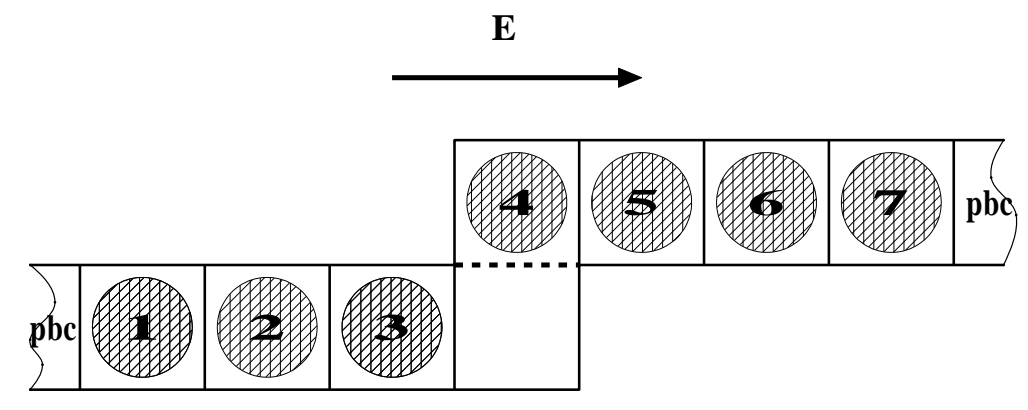

Figure 2: Translational invariance is broken by introducing a kink in the checkerboard (lattice).The jumps across the kink (dashed line) obey different rates, i.e., $q$ and $q^{\prime}$, independently of the particle involved. If the particles carry charges, then in the case of an external electric field $\mathbf{E}$ the jumps across the kink are not biased.

checkerboard (and the DB), as the hole wanders.

Fixing our reference frame to the string of particles, which turns out to be mathematically convenient, we see that the DB 'moves', if and only if the hole passes through it. Further, the jump rates from site $n$ to $n \pm 1$ are not always $W_{n \pm 1, n}$, but take on the value $q$ or $q^{\prime}$ whenever the jump is across the DB. Naively, one may expect that an analytic solution is impossible, given the seemingly time-dependent rates. Nevertheless, we are able to find a solution. The key observation is the existence of a specific linear combination of the positions of the hole and the DB, with which the system of Master equations can be rewritten in a form describing the hopping of a single random walker (called quasiwalker) on a chain of length $N(N+1)$. Exploiting the results of Derrida, the probability distribution, velocity and diffusion constants of the quasi-walker, as well as those for the hole and the DB, can be explicitly computed.

An immediate application for the model with directional impurity is a generalized asymmetric exclusion process (ASEP) [2], [3]. Consider the pieces as charged particles. More precisely, let the particles be charged with $Q_{n}$ and let us apply an external electric field $\mathbf{E}$ pointing along the chain (See Fig.2). Neglecting the interaction among charges, the hopping rates are solely determined by the influence of $\mathbf{E}$ on the charges. Away from the kink, these rates would be biased, due to E. However, across the kink (or DB), particle hops are perpendicular to the external field, so that the corresponding probabilities ( $q$ and $\left.q^{\prime}\right)$ are not biased by $\mathbf{E}$.

Another application, which is also the physical motivation of our model, comes from the connection to the reptation model of Duke-Rubinstein for gel electrophoresis [5]. In Ref. [6] we introduced a model for electrophoresis of polymers with impurities, i.e., polymers having segments (reptons) insensitive to an external electric field $E$. We showed that the effect of a single impurity is equivalent to having a single kink in the ASEP above. The drift velocity of the polymer chain, being related in this model to that of our random walker, is therefore directly affected. The details of this application, which is a particular case of our model, are presented in the last section of this paper.

The structure of the paper is as follows: in Sect. 2 we provide a precise formulation for 
the model with one mobile directional impurity, at the level of Master equations. Here, we show that the structure of these equations leads us to a natural definition of a new entity, namely, the quasi-walker, which stands for the hole-DB couple. A full solution for the steady state probability distribution is then given. From that, the expressions for the velocity and diffusion constant (in the steady state) of this quasi-walker are derived. Section 3 is devoted to a detailed description of the original system in terms of the quasi-walker. Using a technique from the theory of random walks on rings, see [2], [7] (and referred as replication method by us), the expressions for the velocity and diffusion constant for the hole and the DB (with respect to the chain) are obtained. The remaining sections deal with special cases and applications.

\section{Definition of the model and the quasi-walker.}

In this section, we will present another representation of our model which is equivalent to, but simpler than, that shown in Fig. 2. Though there are two moving objects (the hole and the defect bond), the motion of the DB is completely determined by the dynamics of the hole, so that there is, effectively, only one degree of freedom. This single co-ordinate is then associated with the quasi-walker.

Instead of a chain on a kinked checkerboard, our system can be redrawn as in Fig.3a)d). Here, we have our fixed reference frame to be that of the particle chain, arbitrarily choosing one particle as the "first" in the labeling $1,2, \ldots, N$. Representing the particles (hole) by solid (open) circles, we see that the hole is located in between the particles, i.e., at positions denoted by short vertical lines. The DB (which is the kink in Fig.2) can also be thought of a "wanderer" amongst the particles. We denote its position by a long vertical line. The only complication arises when the hole comes "in contact" with the DB, i.e., when both of them are located between two successive particles. Then, there are two distinct states of the system, depending on which side of the DB we find the hole. As a result, we are forced to draw two short vertical lines on either side of the DB.

To keep track of these positions we introduce the following variables:

- Associate the position of the DB with $b$, i.e., when the defect bond is between particles $b$ and $b+1$. Thus, $b \in[1, N]$.

- Let $m$ denote the position of the hole relative to the DB. Since the hole occupies a square in the checkerboard, it is clear that this square can be on either side of the DB (shown by solid line in Fig.2). Thus, $m$ assumes one more value than $b$. We choose $m \in[0, N]$ and let $m=0$ and $N$ be associated with the positions next to the DB (See Fig.3).

For clarity, let us illustrate the dynamics of the hole near the DB. There are 4 representations of the same piece of chain at consecutive time steps. In the first (Fig.3a), the hole is at $m=N-1$ and the bond is at $b$. In the next step, the hole interchanges with particle $b$, arriving at $m=N$ (Fig.3b). Suppose it next interchanges with particle $b+1$. In the checkerboard, this would be an exchange "across the DB", so that the DB will now be found between particles $b+1$ and $b+2$. At the same time, the hole will be "on the other side of the DB". Thus, both the hole and the DB arrive at new positions (Fig.3c). Finally, 

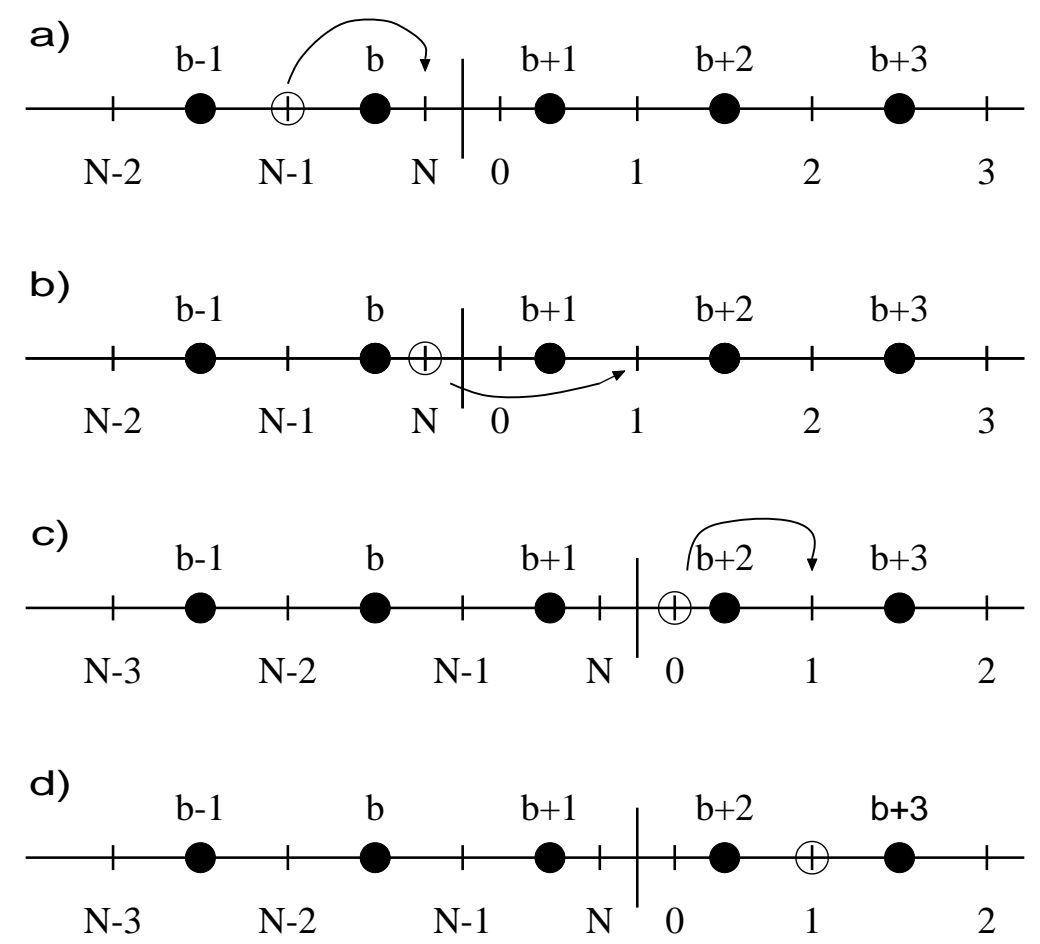

Figure 3: An equivalent representation of the model from Fig. 2. The solid circles represent particles. The short vertical lines mark the possible positions of the hole represented by an open circle. The DB is shown as a long, solid, vertical line. As the hole passes through the DB, the latter encounters a one-step shift in the same direction, a)-d).

according to our scheme, we must "relabel" $m$ accordingly to the value $m=0$. In the last frame, the hole moves further to the right. The DB is not affected, so that only $m$ increases to 1 (Fig.3d). Of course, if the hole moves to the left, then we must reverse the previous process. Let us emphasize that, in the single move between Figs.3b and $3 \mathrm{c}, b$ increments by 1 while $m$ drops from $N$ to 0 . These rules can easily be verified by playing on the checkerboard.

\subsection{The system of Master equations}

The full probabilistic information about our system is contained in $P(b, m ; t)$, the probability to find the DB and the hole at $b$ and $m$, respectively, at time $t$. The time evolution of $P$ will be controlled by the jump rates of the hole, defined in rules 1)-5) above: $W_{i, j}, q$, and $q^{\prime}$. Recall that the subscripts on $W$ refer to the positions of the hole being between particles $i$ and $i+1$, etc. In terms of our variables $b$ and $m$, this position is clearly just $b+m$, modulo $N$, since our chain is a closed one of $N$ particles. Thus, it is natural to introduce the notation:

$$
W_{m, m^{\prime}}^{b} \equiv W_{b+m, b+m^{\prime}}
$$


with the understanding:

$$
W_{i+N, j+N}=W_{i, j}
$$

Since the hole can only exchange with its neighboring particles, the restriction $\left|m-m^{\prime}\right|=1$ applies in (11). Combining these remarks and following the special rules associated with the DB (Fig.3), the evolution of $P$ is given in terms of a system of Master equations:

$$
\begin{aligned}
m=0: \quad \partial_{t} P(b, 0)= & P(b-1, N) q+P(b, 1) W_{0,1}^{b} \\
& -P(b, 0)\left(q^{\prime}+W_{1,0}^{b}\right) \\
m \neq 0, N: \quad \partial_{t} P(b, m)= & P(b, m-1) W_{m, m-1}^{b}+P(b, m+1) W_{m, m+1}^{b} \\
& -P(b, m)\left(W_{m-1, m}^{b}+W_{m+1, m}^{b}\right) \\
m=N: \quad \partial_{t} P(b, N)= & P(b, N-1) W_{N, N-1}^{b}+P(b+1,0) q^{\prime} \\
& -P(b, N)\left(W_{N-1, N}^{b}+q\right)
\end{aligned}
$$

where we have suppressed $t$ for simplicity.

\section{$2.2 \quad$ The steady state limit}

One of our central interests is the steady state limit. Assuming that it exists, let us define

$$
X_{m}^{b} \equiv \lim _{t \rightarrow \infty} P(b, m ; t)
$$

Then, from (3), these satisfy the following set of linear equations:

$$
\begin{aligned}
m=0: & X_{1}^{b} W_{0,1}^{b}+X_{N}^{b-1} q-X_{0}^{b}\left(q^{\prime}+W_{1,0}^{b}\right)=0 \\
m \neq 0, N: & X_{m+1}^{b} W_{m, m+1}^{b}+X_{m-1}^{b} W_{m, m-1}^{b}-X_{m}^{b}\left(W_{m-1, m}^{b}+W_{m+1, m}^{b}\right)=0 \\
m=N: & X_{N-1}^{b} W_{N, N-1}^{b}+X_{0}^{b+1} q^{\prime}-X_{N}^{b}\left(q+W_{N-1, N}^{b}\right)=0
\end{aligned}
$$

These equations can be solved by introducing the steady state probability current densities:

$$
c_{m}^{b} \equiv X_{m}^{b} W_{m-1, m}^{b}-X_{m-1}^{b} W_{m, m-1}^{b}
$$

Now, one can rewrite (5) as:

$$
\begin{aligned}
m=0: & c_{1}^{b}=X_{0}^{b} q^{\prime}-X_{N}^{b-1} q, \\
m \neq 0, N: & c_{m+1}^{b}=c_{m}^{b}, \\
m=N: & c_{N}^{b}=X_{0}^{b+1} q^{\prime}-X_{N}^{b} q .
\end{aligned}
$$


From (8), we find

$$
c_{N}^{b}=c_{N-1}^{b}=\ldots=c_{2}^{b}=c_{1}^{b}
$$

Examining the right hand sides of (7) and (9), we can go one step further:

$$
c_{N}^{b}=c_{1}^{b+1}
$$

In other words, none of these $c$ 's depend on $b$ or $m$, so that we might as well write

$$
c_{m}^{b}=c
$$

This equation can be easily interpreted as: the probability current density in the steady state is a constant. This result is typical for systems with a periodic, one dimensional configuration space, where the current has only a single component and can be fixed by the conservation law. Since we have two variables $(b, m)$, we may regard our configuration space as "two dimensional", so that (12) is perhaps surprising. The resolution is best displayed by explicitly writing the entire system (7, 8, 9) using (12), as in Fig.4. Note that, between the lowest and highest equations, we have the periodic boundary condition (2), i.e., $X_{N}^{0} \equiv X_{N}^{N}$, and $X_{0}^{N+1} \equiv X_{0}^{1}$. Thus, the whole system of equations can be regarded as those for a single, periodic chain. Explicitly, we introduce:

$$
\bar{X}_{k} \equiv X_{m}^{b}, \quad b \in[1, N], m \in[0, N],
$$

where

$$
k \equiv 1+(b-1)(N+1)+m
$$

simply numbers the equations of Fig.4 starting from the bottom. Thus, $k \in[1, M]$, with

$$
M=N(N+1)
$$

Observe that $k$ uniquely determines the pair $(b, m)$ and vice-versa:

$$
\begin{aligned}
b & =\left[\frac{k-1}{N+1}\right]+1 \quad \text { and } \\
m & =(k-1) \bmod (N+1),
\end{aligned}
$$


where $[x]$ represents the integer part of the real number $x$. To display the structure of this single periodic chain more clearly, let us introduce:

$$
\bar{W}_{k, k-1} \equiv W_{m, m-1}^{b} \quad \text { and } \quad \bar{W}_{k-1, k} \equiv W_{m-1, m}^{b} \quad
$$

for $m \in[1, N]$. For $m=0$, we first define

$$
k_{b} \equiv 1+(b-1)(N+1)
$$

Referring to (7, 9), we see that

$$
\bar{W}_{k_{b}, k_{b}-1}=q \text { and } \bar{W}_{k_{b}-1, k_{b}}=q^{\prime}
$$

for all $b$. Finally, to stress the $M$-periodicity of these rates, we write

$$
\bar{W}_{k+M, l+M}=\bar{W}_{k, l}
$$

With this notation the system from Fig.4 reads:

$$
\bar{X}_{k} \bar{W}_{k-1, k}-\bar{X}_{k-1} \bar{W}_{k, k-1}=c, \quad k \in[1, M] .
$$

Recast in the form of (22), our system is exactly the one studied by Derrida, [2]. The only difference is that, in our case, the $\bar{X}_{k}$ stands for the steady state of the "defect bondhole" pair, as opposed to a single particle as in [2]. We will refer to this new entity as a "quasi-walker", and treat it as a single random walker on a chain of length $M$.

Lastly, we remark that not only the steady state limit can be reduced to the case in [2], but also the full time dependence of our system (3) can be mapped. Defining

$$
\bar{P}_{k}(t) \equiv P(b, m ; t)
$$

the system of Master equations (3) can be written as:

$$
\partial_{t} \bar{P}_{k}=\bar{P}_{k+1} \bar{W}_{k, k+1}+\bar{P}_{k-1} \bar{W}_{k, k-1}-\bar{P}_{k}\left(\bar{W}_{k+1, k}+\bar{W}_{k-1, k}\right)
$$

\subsection{Application of Derrida's results to the quasi-walker.}

In this paragraph we apply directly the results from [2] to obtain the steady state probability distribution, the velocity and diffusion constants of our quasi-walker. As expected, all quantities are rational functions of the jump rates. Thus,

$$
\bar{X}_{k}=\Gamma \bar{r}_{k}
$$




$$
\begin{aligned}
& \mathrm{b}=\mathrm{N}\left\{\begin{array}{c}
\mathrm{m}=\mathrm{N} \\
\mathrm{m}=1
\end{array} \begin{array}{c}
\mathrm{X}_{\mathrm{N}}^{\mathrm{N}} \mathrm{W}_{\mathrm{N}-1, \mathrm{~N}}^{\mathrm{N}}-\mathrm{X}_{\mathrm{N}-1}^{\mathrm{N}} \mathrm{W}_{\mathrm{N}, \mathrm{N}-1}^{\mathrm{N}}=\mathrm{c} \\
\vdots \\
\mathrm{X}_{1}^{\mathrm{N}} \mathrm{W}_{0,1}^{\mathrm{N}}-\mathrm{X}_{0}^{\mathrm{N}} \mathrm{W}_{1,0}^{\mathrm{N}}=\mathrm{c}
\end{array}\right. \\
& \mathrm{m}=0 \quad \mathrm{X}_{0}^{\mathrm{N}} \mathrm{q}, \quad-\mathrm{X}_{\mathrm{N}}^{\mathrm{N}-1} \mathrm{q}=\mathrm{c} \\
& \mathrm{b}=\mathrm{N}-1 \quad \mathrm{~m}=\mathrm{N} \quad \mathrm{X}_{\mathrm{N}}^{\mathrm{N}-1} \mathrm{~W}_{\mathrm{N}-1, \mathrm{~N}}^{\mathrm{N}-1}-\mathrm{X}_{\mathrm{N}-1}^{\mathrm{N}-1} \mathrm{~W}_{\mathrm{N}, \mathrm{N}-1}^{\mathrm{N}-1}=\mathrm{c} \\
& \begin{array}{ll|l}
\mathrm{b}+1 & \mathrm{~m}=1 \quad \mathrm{X}_{1}^{\mathrm{b}+1} \mathrm{~W}_{0,1}^{\mathrm{b}+1}-\mathrm{X}_{0}^{\mathrm{b}+1} \mathrm{~W}_{1,0}^{\mathrm{b}+1}=\mathrm{c}
\end{array} \\
& \mathrm{m}=0 \quad \mathrm{X}_{1}^{\mathrm{b}+1} \mathrm{q}^{\prime} \quad-\mathrm{X}_{\mathrm{N}}^{\mathrm{b}} \mathrm{q}=\mathrm{c} \\
& \mathrm{b}\left\{\begin{array}{c|c}
\mathrm{m}=\mathrm{N} & \mathrm{X}_{\mathrm{N}}^{\mathrm{b}} \mathrm{W}_{\mathrm{N}-1, \mathrm{~N}}^{\mathrm{b}}-\mathrm{X}_{\mathrm{N}-1}^{\mathrm{b}} \mathrm{W}_{\mathrm{N}, \mathrm{N}-1}^{\mathrm{b}}=\mathrm{c} \\
\mathrm{m} & \mathrm{X}_{\mathrm{m}}^{\mathrm{b}} \mathrm{W}_{\mathrm{m}-1, \mathrm{~m}}^{\mathrm{b}}-\mathrm{X}_{\mathrm{m}-1}^{\mathrm{b}} \mathrm{W}_{\mathrm{m}, \mathrm{m}-1}^{\mathrm{b}}=\mathrm{c} \\
\mathrm{m}=1 & \vdots \\
\mathrm{X}_{1}^{\mathrm{b}} \mathrm{W}_{0,1}^{\mathrm{b}}-\mathrm{X}_{0}^{\mathrm{b}} \mathrm{W}_{1,0}^{\mathrm{b}}=\mathrm{c}
\end{array}\right. \\
& \mathrm{m}=0 \quad \mathrm{X}_{0}^{\mathrm{b}} \mathrm{q}, \quad-\mathrm{X}_{\mathrm{N}}^{\mathrm{b}-1} \mathrm{q}=\mathrm{c} \\
& \mathrm{m}=\mathrm{N} \quad \mathrm{X}_{\mathrm{N}}^{\mathrm{b}-1} \mathrm{~W}_{\mathrm{N}-1, \mathrm{~N}}^{\mathrm{b}-1}-\mathrm{X}_{\mathrm{N}-1}^{\mathrm{b}-1} \mathrm{~W}_{\mathrm{N}, \mathrm{N}-1}^{\mathrm{b}-1}=\mathrm{c} \\
& \mathrm{b}=1\left\{\begin{array}{l|ll}
\mathrm{m}=\mathrm{N} & \mathrm{X}_{\mathrm{N}}^{1} \mathrm{~W}_{\mathrm{N}-1, \mathrm{~N}}^{1}-\mathrm{X}_{\mathrm{N}-1}^{1} \mathrm{~W}_{\mathrm{N}, \mathrm{N}-1}^{1}=\mathrm{c} \\
\mathrm{m}=1 & \mathrm{X}_{1}^{1} \mathrm{~W}_{0,1}^{1}-\mathrm{X}_{0}^{1} \mathrm{~W}_{1,0}^{1}=\mathrm{c} \\
\mathrm{m}=0 & \mathrm{X}_{0}^{1} \mathrm{q}^{\prime}-\mathrm{X}_{\mathrm{N}}^{\mathrm{N}} \mathrm{C} & =\mathrm{c}
\end{array}\right.
\end{aligned}
$$

Figure 4: The rewritten system. $b$ labels the blocks, $m$ the corresponding equations. 
where

$$
\bar{r}_{k} \equiv \frac{1}{\bar{W}_{k+1, k}}\left[1+\sum_{i=1}^{M-1} \prod_{j=1}^{i} \frac{\bar{W}_{k+j-1, k+j}}{\bar{W}_{k+j+1, k+j}}\right],
$$

and $\Gamma$ is a normalization constant:

$$
\Gamma=\left(\sum_{k=1}^{M} \bar{r}_{k}\right)^{-1}
$$

The velocity and the diffusion constant are given, respectively, by:

$$
V=\Gamma M\left[1-\prod_{k=1}^{M} \frac{\bar{W}_{k, k+1}}{\bar{W}_{k+1, k}}\right]
$$

and

$$
D=\Gamma^{2}\left[V \sum_{k=1}^{M} \bar{u}_{k} \sum_{i=1}^{M} i \bar{r}_{k+i}+M \sum_{k=1}^{M} \bar{W}_{k+1, k} \bar{u}_{k} \bar{r}_{k}\right]-V \frac{M+2}{2}
$$

where

$$
\bar{u}_{k} \equiv \frac{1}{\bar{W}_{k+1, k}}\left[1+\sum_{i=1}^{M-1} \prod_{j=1}^{i} \frac{\bar{W}_{k-j, k+1-j}}{\bar{W}_{k+1-j, k-j}}\right]
$$

While it is straightforward to arrive at (22-27), a subtle process is involved in finding (28,29). Before proceeding, let us review this method [2], which we call "replication". Instead of studying a finite chain with periodic boundary conditions, replicate it into an infinite, periodic chain. Thus, we allow, for example, the indices $i, j$ in $\bar{W}_{i, j}$ to be any integer, together with the periodicity constraint, (21). Similarly, the distribution $\bar{P}_{k}(t)$ in (23) is now defined for all $k \in[-\infty, \infty]$, and satisfies (24) with (21). To compute the steady state quantities defined for the original chain, we must sum over all equivalent sites on the replicated chain. On the other hand, the velocity and diffusion constants can be found from the first and second order moments of $\bar{P}_{k}$. Introducing

$$
x(t)=\sum_{k=-\infty}^{\infty} k \bar{P}_{k}(t) \text { and } x_{2}(t)=\sum_{k=-\infty}^{\infty} k^{2} \bar{P}_{k}(t)
$$

the velocity is naturally

$$
V=\lim _{t \rightarrow \infty} \frac{d}{d t} x(t)
$$


while the diffusion constant is given by:

$$
D=\lim _{t \rightarrow \infty} \frac{d}{d t}\left[x_{2}(t)-(x(t))^{2}\right]
$$

Note that $x(t)$ is the position of the quasi-walker at time $t$, averaged over all paths. In Sect. 3 we present in more detail an extension of this replication technique to describe the hole and the defect bond separately.

Before proceeding, let us present these results in terms of the original jump rates $W_{i, j}$, $q^{\prime}$ and $q$. For convenience, let us first define the following expressions:

$$
\begin{gathered}
S(b, m) \equiv \sum_{i=1}^{N-m-1} \prod_{l=1}^{i} \frac{W_{b+m+l-1, b+m+l}}{W_{b+m+l+1, b+m+l}}, \quad m \in[0, N-2], \\
S(b, N-1) \equiv 0, \\
T(b, m) \equiv \sum_{i=1}^{m} \prod_{l=1}^{i} \frac{W_{b+m-l, b+m+1-l}}{W_{b+m+1-l, b+m-l}}, \quad m \in[1, N], \\
T(b, 0) \equiv 0 .
\end{gathered}
$$

The last term of (34) and (36) also appear frequently, so that we define:

$$
\begin{gathered}
\lambda_{S}(b, m) \equiv \prod_{l=1}^{N-m-1} \frac{W_{b+m+l-1, b+m+l}}{W_{b+m+l+1, b+m+l}} \quad, \quad m \in[0, N-2], \\
\lambda_{S}(b, N-1) \equiv 0 \quad, \\
\lambda_{T}(b, m) \equiv \prod_{l=1}^{m} \frac{W_{b+m-l, b+m-l+1}}{W_{b+m-l+1, b+m-l}} \quad, \quad m \in[1, N], \\
\lambda_{T}(b, 0) \equiv 1
\end{gathered}
$$

Observe that the above quantities depend on neither $q^{\prime}$ nor $q$, and that the $W$ 's satisfy periodicity (2). As a result, they are also periodic: e.g., $S(b+N, m)=S(b, m)$, etc. 
Finally, let:

$$
\lambda \equiv \prod_{b=1}^{N} \frac{W_{b-1, b}}{W_{b+1, b}}
$$

and

$$
U \equiv 1-\left(\frac{q^{\prime}}{q} \lambda\right)^{N}
$$

After some rather tedious algebra, we arrive at the explicit expressions:

$$
\begin{gathered}
\bar{r}_{k_{b}+m}=\frac{1}{W_{b+m+1, b+m}}\left\{U[1+S(b, m)]+W_{b-1, b} \lambda_{S}(b, m) \bar{r}_{k_{b}+N}\right\}, \\
\bar{u}_{k_{b}+m}=\frac{1}{W_{b+m+1, b+m}}\left\{U[1+T(b, m)]+q^{\prime} \lambda_{T}(b, m) \bar{u}_{k_{b-1}+N}\right\} \\
m \in[0, N-1]
\end{gathered}
$$

where

$$
\bar{r}_{k_{b}+N}=\frac{1}{q} \sum_{i=1}^{N}\left(\frac{q^{\prime}}{q} \lambda\right)^{i-1}\left\{1+q^{\prime} \frac{1}{W_{b+i+1, b+i}}[1+S(b+i, 0)]\right\}
$$

and

$$
\bar{u}_{k_{b}+N}=\frac{1}{q} \sum_{i=0}^{N-1}\left(\frac{q^{\prime}}{q} \lambda\right)^{i}[1+T(b-i, N)] .
$$

The normalization constant (27) now takes the form:

$$
\Gamma^{-1}=\sum_{b=1}^{N}\left\{U \sum_{m=0}^{N-1} \frac{1+S(b, m)}{W_{b+m+1, b+m}}+\left[1+W_{b-1, b} \sum_{m=0}^{N-1} \frac{\lambda_{S}(b, m)}{W_{b+m+1, b+m}}\right] \bar{r}_{k_{b}+N} \cdot\right\} .
$$

From (22), we see that the current $c$ can be related [2] to the velocity of the quasi-walker via :

$$
c=-\frac{V}{M}
$$


with $V$ given by (28). Verifying

$$
\prod_{k=1}^{M} \frac{\bar{W}_{k, k+1}}{\bar{W}_{k+1, k}}=\left(\frac{q^{\prime}}{q} \lambda\right)^{N},
$$

we have:

$$
V=\Gamma M U
$$

In other words, $U$ from (43) contains the essential information on the velocity $V$. Further, it indicates whether our system satisfies detailed balance or not, i.e., if we have an equilibrium or a non-equilibrium steady state. The former is the case if and only if $c=0$, so that both $U$ and $V$ vanishes. The condition to stop the motion can be summarized in a simple equation:

$$
q / q^{\prime}=\lambda
$$

Its interpretation is clear. The left hand side is the bias in the jump rate across the defect bond, while the right hand side represents the bias in the opposite direction across the entire chain of particles. When these balance, we have equilibrium.

In the next section, we will see that the expression (51) for the velocity of the quasiwalker plays a central role in determining the velocities of the DB $\left(V_{b o n d}\right)$ and the hole $\left(V_{\text {hole }}\right)$ with respect to the chain, as well as the relative velocity between the two $\left(V_{\text {rel }}\right)$. In particular, an extension of the method of replicating a finite closed chain will be shown in the derivation of these quantities. Following this method we also can derive the diffusion constants for the bond and the hole $D_{\text {bond }}$ and $D_{\text {hole }}$ in the same steady state limit.

\section{Replicating the finite, closed chain}

In a finite, closed chain, a hole will visit the same site infinitely many times in the $t \rightarrow \infty$ limit. As a result, it is not easy to find asymptotic properties like steady state velocities and diffusion constants. To circumvent these difficulties, the method used (see for e.g. [2], [7]) is to replicate the closed chain into an infinite, periodic chain (with periodic jumps rates). Since there are infinitely many sites, though many are "equivalent", this infinite chain greatly facilitates the computation of asymptotic quantities. Here, we will generalize this method and arrive at expressions for the velocities and diffusion constants of both the DB and the hole. The extra complication in our case is that, instead of having one random walker, we have two moving objects: the hole and the defect bond. Therefore, we have to define our replicated model carefully so as not to lose any physical information about the finite, closed chain. 

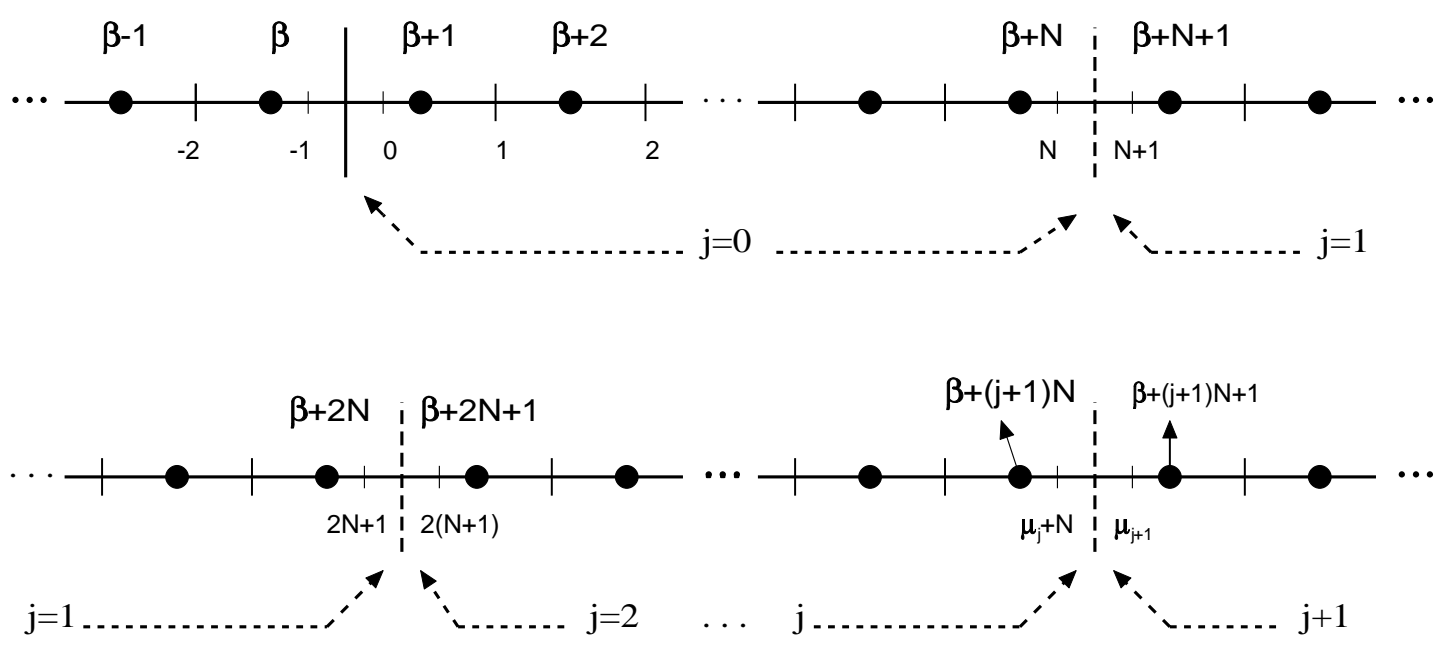

Figure 5: The replicated, infinite chain. The long, solid vertical line represents the DB's position while the long, dashed vertical ones show its images. $j$ labels the number of complete blocks (containing $N$ particles) away from the DB.

On the infinite chain, the position of both the hole and the DB can take arbitrary values, so that they can be found with any distance in between. Let us define the position of the DB, relative to some arbitrarily chosen "origin", to be $\beta$ and the distance from the bond to the hole to be $\mu$. Analogous to $b$ and $m$, these differ in that $\beta, \mu \in[-\infty, \infty]$.

Now, the dynamical rules associated with these are not so simple, however, since they must reflect the fact that, on the closed finite chain, the DB "advances" by a step each time the hole moves forward $N$ steps. To simulate these advances, we introduce an infinite set of "images" of the bond, located at multiples of $N$ from the DB. These images serve as "condition lines" for the motion of the DB itself, i.e., whenever the hole passes through one of these images, the $\beta$ changes by unity. Of course, in accordance with the idea of an image, all the images also move correspondingly. In this way, though the two objects can be arbitrarily far apart on the replicated chain, the properties, or the structure, of the original chain are replicated faithfully. Referring to Fig.5, it is clear that there are unique integers $i$ and $j$, such that

$$
\beta=b+i N \quad \text { and } \quad \mu=m+j(N+1) \quad .
$$

Note that, as expected, $b$ is just $\beta \bmod (N)$. Now, $j$ represents the number of complete replicas of the original chain lying between the DB and the hole, while $m$ is just the distance, as in the previous section, from the hole to the nearest bond-image to its left. 
Let us caution the reader on an extra complication associated with the second equation in (53). The reason for the factor $(N+1)$ lies in our having to distinguish between a hole immediately to the left/right of all the images of the DB. Therefore $\mu$ represents the number of states or positions between the bond and the hole. However, the "actual distance", in terms of number of particles (or units of lattice constant), between the DB and the hole is only $m+j N=\mu-j$ (see Fig.5). In other words, while it is necessary to associate two positions with each image for the hole, there is only one "lattice spacing". This subtle distinction will be crucial when we compute the velocity and diffusion constants, both of which must be based on the physical distance. In particular, the distance of the hole from the origin of the infinite chain is simply $\beta+\mu-j$.

To distinguish the statistical mechanics of the replicated chain from the finite one, we will use notation $\tilde{P}(\beta, \mu ; t)$ for the probability distribution. To write down the Master

equations for $\tilde{P}$, based on the original set (3), we first transcribe $\tilde{W}_{\mu, \mu^{\prime}}^{\beta}$, the transition probability for hole-jumps on the replicated chain. Using the periodicity property (2), we see that these are simply given by

$$
\tilde{W}_{\mu, \mu^{\prime}}^{\beta}=W_{m, m^{\prime}}^{b}
$$

With the notation $\mu_{j} \equiv j(N+1)$, and suppressing $t$, we define the currents

$$
\begin{array}{cl}
m \neq 0: & \tilde{\mathcal{J}}_{\mu}^{\beta}(\tilde{P}) \equiv \tilde{P}(\beta, \mu) \tilde{W}_{\mu-1, \mu}^{\beta}-\tilde{P}(\beta, \mu-1) \tilde{W}_{\mu, \mu-1}^{\beta}, \\
m=0: & \tilde{\mathcal{J}}_{\mu_{j}}^{\beta}(\tilde{P}) \equiv \tilde{P}\left(\beta, \mu_{j}\right) q^{\prime}-\tilde{P}\left(\beta-1, \mu_{j-1}+N\right) q .
\end{array}
$$

In terms of these, the system of Master equations for $\tilde{P}(\beta, \mu)$ reads as:

$$
\begin{aligned}
m=0: & \partial_{t} \tilde{P}\left(\beta, \mu_{j}\right) & =\tilde{\mathcal{J}}_{\mu_{j}+1}^{\beta}(\tilde{P})-\tilde{\mathcal{J}}_{\mu_{j}}^{\beta}(\tilde{P}) \\
m \neq 0, N: & \partial_{t} \tilde{P}(\beta, \mu) & =\widetilde{\mathcal{J}}_{\mu+1}^{\beta}(\tilde{P})-\tilde{\mathcal{J}}_{\mu}^{\beta}(\tilde{P}) \\
m=N: & \partial_{t} \tilde{P}\left(\beta, \mu_{j}+N\right) & =\widetilde{\mathcal{J}}_{\mu_{j+1}}^{\beta+1}(\tilde{P})-\tilde{\mathcal{J}}_{\mu_{j}+N}^{\beta}(\tilde{P}) .
\end{aligned}
$$

\subsection{Positions, velocities and diffusion constants}

To reach our goal of computing velocities, etc., we need some notion of positions. For that, we introduce the following first moments of $\tilde{P}$ :

$$
\langle\beta(t)\rangle=\sum_{\mu=-\infty}^{\infty} \sum_{\beta=-\infty}^{\infty} \beta \tilde{P}(\beta, \mu)
$$




$$
\begin{aligned}
& \langle\mu(t)\rangle=\sum_{\mu=-\infty}^{\infty} \sum_{\beta=-\infty}^{\infty} \mu \tilde{P}(\beta, \mu) \\
& \langle j(t)\rangle=\sum_{\mu=-\infty}^{\infty} \sum_{\beta=-\infty}^{\infty} j \tilde{P}(\beta, \mu) .
\end{aligned}
$$

Is easy to see that, the average position for the $\mathrm{DB}$ is just $\langle\beta(t)\rangle$. For the average distance between the hole and the DB, we define

$$
\langle\delta(t)\rangle=\langle\mu(t)\rangle-\langle j(t)\rangle
$$

so that the average position of the hole is

$$
\langle h(t)\rangle \equiv\langle\beta(t)\rangle+\langle\delta(t)\rangle=\langle\beta(t)\rangle+\langle\mu(t)\rangle-\langle j(t)\rangle .
$$

The time derivatives of the moments defined above can be used to compute velocities:

$$
\begin{aligned}
v_{\text {bond }}(t) & \equiv \frac{d}{d t}\langle\beta(t)\rangle, \\
v_{\mu}(t) & \equiv \frac{d}{d t}\langle\mu(t)\rangle, \\
v_{j}(t) & \equiv \frac{d}{d t}\langle j(t)\rangle .
\end{aligned}
$$

The first clearly represents the average velocity, with respect to the chain, of the DB. Naively, the second can be thought of as the velocity of the hole relative to the bond on this infinite chain, since $\mu$ plays the role of $m$ here. However, in view of the distinction between $\mu$ and the "actual distance" $\mu-j$, we emphasize that the "actual velocity" of the hole relative to the bond, is

$$
v_{r e l}(t) \equiv \frac{d}{d t}\langle\delta(t)\rangle=v_{\mu}(t)-v_{j}(t)
$$

while the hole's velocity, relative to the chain itself is 


$$
v_{\text {hole }} \equiv \frac{d}{d t}\langle h(t)\rangle=v_{\text {bond }}(t)+v_{\text {rel }}(t)
$$

The diffusion constants for the DB and for the hole can be found similarly:

$$
\begin{aligned}
& d_{\text {bond }}(t)=\frac{1}{2}\left[\frac{d}{d t}\left(\left\langle\beta^{2}(t)\right\rangle\right)-\frac{d}{d t}\left(\langle\beta(t)\rangle^{2}\right)\right], \\
& d_{\text {hole }}(t)=\frac{1}{2}\left[\frac{d}{d t}\left(\left\langle h^{2}(t)\right\rangle\right)-\frac{d}{d t}\left(\langle h(t)\rangle^{2}\right)\right],
\end{aligned}
$$

where

$$
\begin{gathered}
\frac{d}{d t}\left[\left\langle\beta^{2}(t)\right\rangle\right]=\sum_{\mu=-\infty}^{\infty} \sum_{\beta=-\infty}^{\infty} \beta^{2} \partial_{t} \tilde{P}(\beta, \mu), \\
\frac{d}{d t}\left[\left\langle h^{2}(t)\right\rangle\right]=\sum_{\mu=-\infty}^{\infty} \sum_{\beta=-\infty}^{\infty}(\beta+\mu-j)^{2} \partial_{t} \tilde{P}(\beta, \mu) .
\end{gathered}
$$

Needless to say, all the six second order moments are needed (all quadratic combinations of $\beta, \mu$ and $j$ ). In this paper, we will show explicit calculations for the first order moments and velocities only, since the computations for the second order moments are considerably lengthier yet providing no additional insight.

\subsection{Reductions to the finite, closed chain}

As the original system consists of only a finite, closed chain, it is important to make the connection between the replicated, infinite chain above and the finite one. The simplest way is to note that $i$ and $j$ play the role of replica indices. Thus, we have:

$$
P(b, m ; t)=\sum_{i=-\infty}^{\infty} \sum_{j=-\infty}^{\infty} \tilde{P}(\beta, \mu ; t)
$$

To facilitate the discussion of the moments (57-59), we define

$$
\phi(b, m)=\sum_{i=-\infty}^{\infty} \sum_{j=-\infty}^{\infty} \beta \tilde{P}(\beta, \mu),
$$




$$
\psi(b, m)=\sum_{i=-\infty}^{\infty} \sum_{j=-\infty}^{\infty} \mu \tilde{P}(\beta, \mu),
$$

so that the positions and velocities are given by

$$
\begin{gathered}
\langle\beta(t)\rangle=\sum_{b=1}^{N} \sum_{m=0}^{N} \phi(b, m) \\
\langle\mu(t)\rangle=\sum_{b=1}^{N} \sum_{m=0}^{N} \psi(b, m) \\
\langle j(t)\rangle=\frac{1}{N+1} \sum_{b=1}^{N} \sum_{m=0}^{N}[\psi(b, m)-m P(b, m)]=\frac{1}{N+1}\left[\langle\mu(t)\rangle-\sum_{b=1}^{N} \sum_{m=0}^{N} m P(b, m)\right] \\
v_{\text {bond }}(t)=\sum_{b=1}^{N} \sum_{m=0}^{N} \partial_{t} \phi(b, m) \\
v_{\mu}(t)=\sum_{b=1}^{N} \sum_{m=0}^{N} \partial_{t} \psi(b, m)
\end{gathered}
$$

and

$$
v_{j}(t)=\frac{1}{N+1}\left[v_{\mu}(t)-\sum_{b=1}^{N} \sum_{m=0}^{N} m \partial_{t} P(b, m)\right] .
$$

To compute (77.79) and the time derivatives of the six second order moments, we should use the Master equation for $P(b, m)$ to eliminate $\partial_{t} P$ in favor of the currents and derive similar equations for $\phi(b, m)$ and $\psi(b, m)$. Not surprisingly, these equations will comprise of current-like terms. Letting $\alpha$ be any of the quantities $P, \phi$ or $\psi$, we define: 


$$
\begin{array}{ll}
m=0: & \mathcal{J}_{0}^{b}(\alpha) \equiv \alpha(b, 0) q^{\prime}-\alpha(b-1, N) q \\
m \neq 0: & \mathcal{J}_{m}^{b}(\alpha) \equiv \alpha(b, m) W_{m-1, m}^{b}-\alpha(b, m-1) W_{m, m-1}^{b}
\end{array}
$$

and note that these represent the net probability current density from state $(b, 0)$ to $(b-1, N)$ for $m=0$ and $(b, m)$ to $(b, m-1)$ for $m \neq 0$, respectively. Using these, the definitions (71- 73 ) and the Master equation for $\tilde{P}$, we obtain:

$$
\begin{aligned}
& m=0: \quad \partial_{t} P(b, 0)=\mathcal{J}_{1}^{b}(P)-\mathcal{J}_{0}^{b}(P) \\
& 0<m<N: \quad \partial_{t} P(b, m)=\mathcal{J}_{m+1}^{b}(P)-\mathcal{J}_{m}^{b}(P) \quad \text {, } \\
& m=N: \quad \partial_{t} P(b, N)=\mathcal{J}_{0}^{b+1}(P)-\mathcal{J}_{N}^{b}(P) \\
& m=0: \quad \partial_{t} \phi(b, 0)=\mathcal{J}_{1}^{b}(\phi)-\mathcal{J}_{0}^{b}(\phi)+P(b-1, N) q \\
& 0<m<N: \quad \partial_{t} \phi(b, m)=\mathcal{J}_{m+1}^{b}(\phi)-\mathcal{J}_{m}^{b}(\phi) \quad \text {, } \\
& m=N: \quad \partial_{t} \phi(b, N)=\mathcal{J}_{0}^{b+1}(\phi)-\mathcal{J}_{N}^{b}(\phi)-P(b+1,0) q^{\prime} \\
& m=0: \quad \partial_{t} \psi(b, 0)=\mathcal{J}_{1}^{b}(\psi)-\mathcal{J}_{0}^{b}(\psi)+P(b-1, N) q-P(b, 1) W_{0,1}^{b} \\
& 0<m<N: \quad \partial_{t} \psi(b, m)=\mathcal{J}_{m+1}^{b}(\psi)-\mathcal{J}_{m}^{b}(\psi)+P(b, m-1) W_{m, m-1}^{b}- \\
& P(b, m+1) W_{m, m+1}^{b} \\
& m=N: \quad \partial_{t} \psi(b, N)=\mathcal{J}_{0}^{b+1}(\psi)-\mathcal{J}_{N}^{b}(\psi)+P(b, N-1) W_{N, N-1}^{b}- \\
& P(b+1,0) q^{\prime}
\end{aligned}
$$

Note that (81) is just (3), as it should be. Now, keeping in mind the periodicity property $P(b+N, m)=P(b, m)$, we may express for the velocities (77- 79) in terms of the probability current-density alone:

$$
\begin{aligned}
v_{\text {bond }}(t) & =-\sum_{b=1}^{N} \mathcal{J}_{0}^{b}(P) \\
v_{\mu}(t) & =-\sum_{b=1}^{N} \sum_{m=0}^{N} \mathcal{J}_{m}^{b}(P) \\
v_{j}(t) & =v_{\text {bond }}(t)
\end{aligned}
$$


The last of these, obtained by explicit computation, can be understood intuitively. Since $j$ measures of the net number of times the hole traverse around the (finite) chain, while the DB moves one step each time the hole crosses it, the displacements in $j$ and the bond are locked. From these, we easily arrive at

$$
v_{r e l}(t)=v_{\mu}(t)-v_{j}(t)=v_{\mu}(t)-v_{b o n d}(t)
$$

and

$$
v_{\text {hole }}(t)=v_{\mu}(t)
$$

The $t$-derivative of the second moments, (69) and (70) can be computed similarly:

$$
\frac{d}{d t}\left(\left\langle\beta^{2}(t)\right\rangle\right)=-2 \sum_{b=1}^{N} \mathcal{J}_{0}^{b}(\phi)+\sum_{b=1}^{N}\left[P(b, N) q+P(b, 0) q^{\prime}\right] .
$$

and

$$
\begin{aligned}
& \frac{d}{d t}\left(\left\langle h^{2}(t)\right\rangle\right)=-2 \sum_{b=1}^{N} \sum_{m=0}^{N} \mathcal{J}_{m}^{b}(\phi)-2 \frac{N}{N+1} \sum_{b=1}^{N} \sum_{m=0}^{N} \mathcal{J}_{m}^{b}(\psi) \\
&-\frac{2}{N+1} \sum_{b=1}^{N} \sum_{m=0}^{N} m \mathcal{J}_{m}^{b}(P)+2 \sum_{b=1}^{N} P(b-1, N) q \\
&+ \sum_{b=1}^{N}\left\{\left[P(b, 0) q^{\prime}+\right.\right. \\
&\left.P(b-1, N) q]+\sum_{m=1}^{N}\left[P(b, m) W_{m-1, m}^{b}+P(b, m-1) W_{m, m-1}^{b}\right]\right\} .
\end{aligned}
$$

Note that these expressions involve sums, as opposed to differences, of terms like $P q$ and $P W$, so that they are not simply related to the currents.

These results are general, applicable for all $t$. Our interest lies in the long time steady state limit, which is the focus of the next subsection.

\subsection{Steady state limit}

In the $t \rightarrow \infty$ limit, we already know that $P(b, m ; t) \rightarrow X_{m}^{b}$ (Section 2.2-3). For the other two, our expectations of the asymptotic behavior lead us to the ansatz:

$$
\begin{aligned}
& \phi(b, m) \rightarrow \omega_{m}^{b} t+\Phi_{m}^{b} \\
& \psi(b, m) \rightarrow \lambda_{m}^{b} t+\Psi_{m}^{b}
\end{aligned} .
$$


To determine the four unknowns, we insert these asymptotic forms into (81- 83) and obtain a systems of equations for them. Letting $\alpha$ denote either $\omega$ or $\lambda$, we have

$$
\begin{aligned}
m=0: & 0 & =\mathcal{J}_{1}^{b}(\alpha)-\mathcal{J}_{0}^{b}(\alpha) \\
m \neq 0, N: & & 0=\mathcal{J}_{m+1}^{b}(\alpha)-\mathcal{J}_{m}^{b}(\alpha) \\
m=N: & 0 & =\mathcal{J}_{0}^{b+1}(\alpha)-\mathcal{J}_{N}^{b}(\alpha)
\end{aligned}
$$

For $\Phi_{m}^{b}$ and $\Psi_{m}^{b}$, we find

$$
\begin{aligned}
m=0: & \omega_{0}^{b}=\mathcal{J}_{1}^{b}(\Phi)-\mathcal{J}_{0}^{b}(\Phi)+X_{N}^{b-1} q \\
m \neq 0, N: & \omega_{m}^{b}=\mathcal{J}_{m+1}^{b}(\Phi)-\mathcal{J}_{m}^{b}(\Phi) \\
m=N: & \omega_{N}^{b}=\mathcal{J}_{0}^{b+1}(\Phi)-\mathcal{J}_{N}^{b}(\Phi)-X_{0}^{b+1} q^{\prime}
\end{aligned}
$$

and

$$
\begin{array}{cl}
m=0: & \lambda_{0}^{b}=\mathcal{J}_{1}^{b}(\Psi)-\mathcal{J}_{0}^{b}(\Psi)+X_{N}^{b-1} q-X_{1}^{b} W_{0,1}^{b} \\
m \neq 0, N: & \lambda_{m}^{b}=\mathcal{J}_{m+1}^{b}(\Psi)-\mathcal{J}_{m}^{b}(\Psi)+X_{m-1}^{b} W_{m, m-1}^{b}-X_{m+1}^{b} W_{m, m+1}^{b} \\
m=N: & \lambda_{N}^{b}=\mathcal{J}_{0}^{b+1}(\Psi)-\mathcal{J}_{N}^{b}(\Psi)+X_{N-1}^{b} W_{N, N-1}^{b}-X_{0}^{b+1} q^{\prime} .
\end{array}
$$

Now, (92) is the same set of equations as $X_{m}^{b}$, so that $\omega_{m}^{b}$ and $\lambda_{m}^{b}$ must be both propoertional to $X_{m}^{b}$. Let us write

$$
\begin{aligned}
& \omega_{m}^{b}=V_{\text {bond }} X_{m}^{b}, \\
& \lambda_{m}^{b}=V_{\mu} X_{m}^{b}
\end{aligned}
$$

where the constants $V_{b o n d}$ and $V_{\mu}$ can be fixed by the normalization condition $\sum_{b=1}^{N} \sum_{m=0}^{N} X_{m}^{b}=1$ :

$$
\begin{aligned}
V_{\text {bond }} & =\sum_{b=1}^{N} \sum_{m=0}^{N} \omega_{m}^{b}, \\
V_{\mu} & =\sum_{b=1}^{N} \sum_{m=0}^{N} \lambda_{m}^{b} .
\end{aligned}
$$

Using (77-79) and (91), we arrive at the meaning of these constants:

$$
\begin{aligned}
V_{\text {bond }} & =\lim _{t \rightarrow \infty} \frac{d}{d t}\langle\beta(t)\rangle=\lim _{t \rightarrow \infty} v_{\text {bond }}(t), \\
V_{\mu} & =\lim _{t \rightarrow \infty} \frac{d}{d t}\langle\mu(t)\rangle=\lim _{t \rightarrow \infty} v_{\mu}(t) .
\end{aligned}
$$


In a similar way, we have $V_{j} \equiv \lim _{t \rightarrow \infty} d\left(\left\langle v_{j(t)}\right\rangle\right) / d t=V_{\text {bond }}, V_{\text {rel }}=V_{\mu}-V_{\text {bond }}$ and $V_{\text {hole }}=V_{\mu}$.

Next, we apply the results from Sect. 2.2-3, namely:

$$
\lim _{t \rightarrow \infty} \mathcal{J}_{m}^{b}(P)=\mathcal{J}_{m}^{b}(X)=c=-\frac{V}{M}
$$

and find, from (84),

$$
\begin{aligned}
V_{\text {bond }} & =-N c=\frac{V}{N+1}, \\
V_{\mu} & =-M c=V, \\
V_{j} & =\frac{V}{N+1}, \\
V_{\text {rel }} & =\frac{N}{N+1} V \\
V_{\text {hole }} & =V .
\end{aligned}
$$

Note that these results can also be obtained, according to (97) and (98), by summing (93) and (94) over $m$ and $b$.

Finally, we turn our attention to the diffusion constants (67- 68). Using the linearity property of $\mathcal{J}_{m}^{b}(\alpha)$ as function of $\alpha$, as well as the expressions (95-96) and (99-100) with (84 85), we verify that terms linear in $t$ cancel. The expressions are somewhat simpler if we used the renumbering scheme (14 19) for the quasi-walker. Accordingly, all quantities of the form $A_{m}^{b}$ are now denoted by $\bar{A}_{k}$, where $k=1, . ., M$. In this notation, the currentdensity type quantities in (80) are summarized in one line:

$$
\overline{\mathcal{J}}_{k}(\bar{A})=\bar{A}_{k} \bar{W}_{k-1, k}-\bar{A}_{k-1} \bar{W}_{k, k-1} \quad, \quad k=1, . ., M
$$

As in the previous section, we can solve these for $\bar{A}_{k}$. If we sum over $k$, an important relation is obtained:

$$
\sum_{k=1}^{M} \bar{A}_{k}=\left(-\frac{\Gamma M}{V}\right) \sum_{k=1}^{M} \bar{u}_{k} \overline{\mathcal{J}}_{k+1}(\bar{A}),
$$

where $\bar{u}_{k}$ is given by (30). Using this notation, the diffusion constants are: 


$$
\begin{aligned}
D_{\text {bond }}= & -\sum_{b=1}^{N} \mathcal{J}_{0}^{b}(\Phi)+\frac{1}{2} \sum_{b=1}^{N}\left(X_{0}^{b} q^{\prime}+X_{N}^{b-1} q\right)-V_{\text {bond }} \sum_{b=1}^{N} \sum_{m=0}^{N} \Phi_{m}^{b} \\
D_{\text {hole }}= & -\sum_{k=1}^{M} \overline{\mathcal{J}}_{k}(\bar{\Phi})-V \sum_{k=1}^{M} \bar{\Phi}_{k}+\sum_{b=1}^{N} \bar{X}_{k_{b}-1} q \\
& +\frac{N}{N+1}\left[-\sum_{k=1}^{M} \overline{\mathcal{J}}_{k}(\bar{\Psi})-V \sum_{k=1}^{M} \bar{\Psi}_{k}+\sum_{k=1}^{M} \bar{X}_{k-1} \bar{W}_{k, k-1}\right] \\
& -\frac{V}{N+1} \sum_{k=1}^{M} m \bar{X}_{k}-\frac{V}{2} \cdot \frac{1}{N+1} .
\end{aligned}
$$

Now, we need to find expressions for $\overline{\mathcal{J}}_{k}(\bar{\Phi})$ and $\overline{\mathcal{J}}_{k}(\bar{\Psi})$. These can be found by solving (93) and (94):

$$
\begin{aligned}
\overline{\mathcal{J}}_{k}(\bar{\Phi}) & =\frac{V}{N+1} \frac{1}{M} \sum_{k^{\prime}=1}^{M} k^{\prime} \bar{X}_{k^{\prime}+k-1}+\frac{V}{N+1} \frac{m}{M} \\
& -\frac{V}{N+1} \frac{1}{M} \sum_{k^{\prime}=1}^{M} k^{\prime} \bar{X}_{k^{\prime}}+\delta_{k, k_{b}} \bar{X}_{k_{b}-1} q+c_{\Phi}
\end{aligned}
$$

and

$$
\overline{\mathcal{J}}_{k}(\bar{\Psi})=\frac{V}{M} \sum_{k^{\prime}=1}^{M} k^{\prime} \bar{X}_{k^{\prime}+k-1}+\bar{X}_{k-1} \bar{W}_{k, k-1}-\frac{V}{M} \sum_{k^{\prime}=1}^{M} k^{\prime} \bar{X}_{k^{\prime}}+c_{\Psi}
$$

where $m=(k-1) \bmod (N+1)$ and $c_{\Phi(\Psi)}$ are constants which will disappear from the final expressions. Using (108) and substituting (111, 112) into (109, 110), we finally arrive at explicit forms for the diffusion constants:

$$
\begin{aligned}
D_{\text {bond }} & =\frac{\Gamma^{2}}{(N+1)^{2}}\left[V \sum_{k=1}^{M} \bar{u}_{k} \sum_{k^{\prime}=1}^{M} k^{\prime} \bar{r}_{k^{\prime}+k}+M \sum_{k=1}^{M} \bar{u}_{k_{b}-1} \bar{r}_{k_{b}-1} q\right] \\
& +\frac{\Gamma V}{(N+1)^{2}} \sum_{k=1}^{M}\left(\bar{u}_{k-1}-\bar{r}_{k}\right) m-\frac{M+2}{2} \frac{V}{(N+1)^{2}}
\end{aligned}
$$

and

$$
D_{\text {hole }}=\Gamma^{2}\left(V \sum_{k=1}^{M} \bar{u}_{k} \sum_{k^{\prime}=1}^{M} k^{\prime} \bar{r}_{k^{\prime}+k}+M \sum_{k=1}^{M} \bar{u}_{k} \bar{r}_{k} \bar{W}_{k+1, k}\right)-V \frac{M+2}{2}
$$




$$
+\Gamma^{2} N \sum_{k=1}^{M}\left[\bar{u}_{k_{b}-1} \bar{r}_{k_{b}-1} q-\bar{u}_{k-1} \bar{r}_{k-1} \bar{W}_{k, k-1}\right]+\frac{\Gamma V}{N+1} \sum_{k=1}^{M}\left(\bar{u}_{k-1}-\bar{r}_{k}\right) m .
$$

By comparing these formulae with that for the quasi-walker, (29), the following simple relations are found:

$$
D_{\text {bond }}=\frac{1}{(N+1)^{2}} D+\frac{1}{N+1} \Delta
$$

and

$$
D_{\text {hole }}=D+\Delta,
$$

where $\Delta$ is

$$
\Delta=\Gamma^{2} N \sum_{k=1}^{M}\left[\bar{u}_{k_{b}-1} \bar{r}_{k_{b}-1} q-\bar{u}_{k-1} \bar{r}_{k-1} \bar{W}_{k, k-1}\right]+\frac{\Gamma V}{N+1} \sum_{k=1}^{M}\left(\bar{u}_{k-1}-\bar{r}_{k}\right) m .
$$

Eliminating $\Delta$, the diffusive properties of the hole, the DB and the quasi-walker can be related:

$$
D_{\text {hole }}=(N+1) D_{\text {bond }}+\frac{N}{N+1} D
$$

These relationships, together with those for the velocities (102 - 106), summarize and confirm our intuitive picture of the asymptotic properties associated with not only the physical objects, the impurity (DB) and the hole, but also the quasi-walker. In the next section, we will analyze some special cases in detail, in order to illustrate the use of these results.

\section{Special cases}

In this Section we consider some simple, yet interesting, cases. The explicit expressions for the velocities and diffusion constants will be transparent, painting a clear picture of the physical situation. We will restrict ourselves to two main limits: i) the pure asymmetric and symmetric cases and $i$ ) the random symmetric case. In the former, except for jumps across the defect bond, the particle-hole exchange rates to the left (right) are uniform, given by $W_{\leftarrow}\left(W_{\rightarrow}\right)$. Thus, the term "pure asymmetric case" was coined, while the "pure symmetric case" consists of a further reduction: $W_{\leftarrow}=W_{\rightarrow}$. Across the DB, the exchange rates (of any particle with the hole) are kept general: $q$ and $q^{\prime}$, though we also consider the limit $q=q^{\prime}$ [6]. For case ii), the exchange rate of any particular particle with the hole is randomly chosen, but indepedent of the jump direction - thus the term 
"random symmetric". The random asymmetric case is extremely interesting, being the most general. However, its treatment is quite involved and is beyond the scope of this paper.

As we saw in the preceeding Section, the velocity and diffusion constant of the quasiwalker determines almost all interesting quantities of the system. Therefore, we first give these expressions in the particular cases.

\subsection{Pure asymmetric and symmetric cases}

To be specific, for the asymmetric case, we have, for all $n$ :

$$
W_{n+1, n} \equiv W_{\rightarrow} \quad \text { and } \quad W_{n-1, n} \equiv W_{\leftarrow} \quad
$$

Since all the hopping rates in each direction are identical, the particles in the system are no longer distinguishable. It is meaningless to specify "the location of the DB with respect to the chain." We can just as easily regard the hole as a particle, suffering biased diffusion (if $W_{\leftarrow} \neq W_{\rightarrow}$ ) everywhere except across a specific bond (where the bias is $q-q^{\prime}$ ). The model reduces to a simple driven system with a single blockage [8], so that its properties can be relatively easily understood.

Define

$$
s \equiv W_{\leftarrow} / W_{\rightarrow} \quad,
$$

so that $\lambda=s^{N}$ (from (42)). Using the expressions from Sect. 2.2-3, we then find:

$$
\bar{r}_{k_{b}+m}=\frac{U}{\left(W_{\rightarrow}-W_{\leftarrow}\right)}\left\{1-\lambda s^{-m}+\frac{\lambda s^{-m}}{q-q^{\prime} \lambda}\left[W_{\rightarrow}-W_{\leftarrow}+q^{\prime}(1-\lambda)\right]\right\},
$$

and

$$
\begin{gathered}
\bar{u}_{k_{b}+m}=\frac{U}{\left(W_{\rightarrow}-W_{\leftarrow}\right)}\left[1-s^{m+1}+\frac{q^{\prime} s^{m}}{q-q^{\prime} \lambda}(1-\lambda s)\right], \quad m \in[0, N-1] \\
\bar{u}_{k_{b}+N}=\frac{U}{q-q^{\prime} \lambda} \cdot \frac{1-\lambda s}{1-s} \quad, \quad m=N .
\end{gathered}
$$

where $U$ is defined by (43). The normalization constant now takes the form:

$$
\Gamma^{-1}=\frac{U N}{W_{\rightarrow}-W_{\leftarrow}}\left[N+1+\frac{W_{\rightarrow}-W_{\leftarrow}+q^{\prime}-q}{q-q^{\prime} \lambda} \cdot \frac{1-\lambda s}{1-s}\right] .
$$


Note that, due to the translational invariance of the pure case, the steady state probability distribution $X_{m}^{b}$ is independent of $b$, the DB position. Using (28), we find the quasi-walker's velocity $V$ to be:

$$
V=\left(W_{\rightarrow}-W_{\leftarrow}\right)\left[1+\frac{1}{N+1}\left(1-\frac{q-q^{\prime}}{W_{\rightarrow}-W_{\leftarrow}}\right) \frac{W_{\rightarrow}^{N+1}-W_{\leftarrow}^{N+1}}{q W_{\rightarrow}^{N}-q^{\prime} W_{\leftarrow}^{N}}\right]^{-1}
$$

Since the velocities of the physical objects are directly related to this $V$, it is of interest to point out two appealing aspects of this expression. The effect of the impurity clearly affects $V$ at the level of $O(1 / N)$. Further, it can be completely eliminated by choosing $q^{\prime}=W_{\leftarrow}$ and $q=W_{\rightarrow}$, where $V$ reduces to the expected result: $W_{\rightarrow}-W_{\leftarrow}$. In this limit, the probability distribution in fact becomes uniform, i.e., $X_{m}^{b}=1 / M$.

A similar expression of the diffusion constant $D$ can be given. It is quite cumbersome and not very transparent. So, instead of providing the formula here, let us direct the reader to the general case discussed in the last two Sections. However, it is easy to check that, if we "eliminate" the DB as above, we recover the expected results: $D=\left(W_{\rightarrow}+W_{\leftarrow}\right) / 2$, $\Delta=0, D_{\text {hole }}=D$, and $D_{\text {bond }}=D_{\text {hole }} /(N+1)^{2}$. The first of these was obtained by Derrida [2]. The last equation implies that a referrence point (tagged "defect bond") on the checkerboard diffuses with respect to the string of particles at a rate $N^{-2}$ times slower than the hole itself. In other words, the string of particles, as a whole, has a diffusion constant given by $D_{\text {bond }}$.

The case pointed out in Ref. [6], applicable to the reptation model of a polymer with a single drive-insensitive impurity, is given by $q^{\prime}=q \equiv B$. From (125) we see that the impurity reduces the drift velocity by $O(1 / N)$. For this case, the interesting generalization of our model lies in the study of chains with a fixed fraction of holes and/or impurities.

From the "pure asymmetric case", we can simplify further to the symmetric limit, in which $W_{\rightarrow}=W_{\leftarrow} \equiv W$ with arbitrary $q$ and $q^{\prime}$. The corresponding physical picture is a hole (or a particle) performing simple random walk everywhere except across one bond, where it receives an extra "kick" of strength $q-q^{\prime}$ to the right. Thus, this case can be mapped into simple diffusion with open boundaries, coupled to chemical potentials. Solving the ordinary diffusion equation with unequal densities at the two boundaries is trivial: the result is a linear profile. Indeed, this is reproduced here. Setting $s=\lambda=1$, we find the probability distribution:

$$
X_{m}^{b}=\frac{1}{M} \frac{q(N-m)+q^{\prime} m+W}{\bar{q} N+W}
$$

where $\bar{q}=\left(q+q^{\prime}\right) / 2$ is the average jump rate through the defect bond. The velocity, which is related to the current, also takes a simple form:

$$
V=\frac{q-q^{\prime}}{1+N \frac{\bar{q}}{W}}
$$


However the expression for the diffusion constants remain rather involved. They only simplify with the further restriction: $q=q^{\prime}$ (where clearly the probability distribution is uniform and $V=0$ ). Then

$$
D=q \frac{1+N}{1+N \frac{q}{W}}
$$

with $\Delta=0, D_{\text {hole }}=D$, and $D_{\text {bond }}=D /(N+1)^{2}$.

\subsection{Random symmetric case}

In this part we study the case where the $W$ jump rates are symmetric:

$$
W_{n, j}=W_{j, n}
$$

but otherwise randomly chosen out of some distribution. The rates $q$ and $q^{\prime}$ are also kept in general.

An immediate consequence is $\lambda=1$. Using the expressions (44-47) for $\bar{r}_{k}$ and $\bar{u}_{k}$ and introducing

$$
z \equiv \sum_{l=1}^{N} \frac{1}{W_{l+1, l}}
$$

for convenience, we arrive at:

$$
\bar{r}_{k_{b}+m}=\left[1-\left(\frac{q^{\prime}}{q}\right)^{N}\right]\left[\frac{1+q^{\prime} z}{q-q^{\prime}}+\sum_{i=1}^{N-m} \frac{1}{W_{b+m+i, b+m+i-1}}\right] \quad, m \in[0, N]
$$

and

$$
\begin{gathered}
\bar{u}_{k_{b}+N}=\frac{N+1}{q-q^{\prime}}\left[1-\left(\frac{q^{\prime}}{q}\right)^{N}\right], \\
\bar{u}_{k_{b}+m}=\frac{1}{W_{b+m+1, b+m}}\left[1-\left(\frac{q^{\prime}}{q}\right)^{N}\right] \frac{q(m+1)+q^{\prime}(N-m)}{q-q^{\prime}} .
\end{gathered}
$$

From the normalization constant

$$
\Gamma^{-1}=M\left[1-\left(\frac{q^{\prime}}{q}\right)^{N}\right] \frac{1+\bar{q} z}{q-q^{\prime}},
$$


we obtain the velocity:

$$
V=\frac{q-q^{\prime}}{1+\bar{q} z}
$$

This formulae also displays some intuitively understandable properties, e.g., proportionality to the drive $\left(q-q^{\prime}\right)$ and being of $O(1 / N)$ (through $\left.z \sim O(N)\right)$. As in the previous cases, explicit expressions for the diffusion constants are not particularly illuminating and will not be given here. However, in the totally symmetric case, i.e., $q=q^{\prime}$, there is simplification:

$$
D=q \frac{1+N}{1+z q}
$$

with $\Delta=0, D_{\text {hole }}=D$, and $D_{\text {bond }}=D /(N+1)^{2}$.

If the $W$ 's are drawn from the distribution $\rho(W)$, then the expressions for the velocity and diffusion constants are valid in the thermodynamic limit $N \rightarrow \infty$ with $z / N=$

$\int \rho(W) \frac{d W}{W}$. Finally, we point out the obvious: for a $\delta$ distribution, (135, 136) reduce to (127,128).

\section{Summary and outlook}

In this paper, we study a generalized asymmetric exclusion process (ASEP) [2] to include a directional impurity. The simplest realization consists of a random walker (a hole) on a one dimensional periodic lattice which is otherwise filled with particles. The rates at which the hole exchanges with any particular particle $i(i=1, \ldots, N)$ is arbitrary and direction-dependent (but time-indepedent). In otherwords, the rate of a particle hop to the right, $W_{i+1, i}$, can be different from those of a hop to the left, $W_{i-1, i}$. However, across one special bond (which is fixed in respect to the lattice and not the string of particles), which we call the "defect bond", the rates of particle-hole exchange is fixed at $q$ and $q^{\prime}$, regardless of which particle is involved. In general, the $q$ 's are of course unrelated to the $W$ 's. In this sense, there is an "impurity" in the lattice, associated with the mobility of the walker, which breaks translational invariance.

Though the problem appears to have two degrees of freedom - the positions of the hole and the defect bond - we show that the associated configuration space is, in fact, one-dimensional. There is a subtle coupling of these two objects into a new entity which we label the quasi-walker. To be precise, the location of the bond, $b$, and the bond-hole separation, $m$, can be combined into a single variable $k \equiv 1+(b-1)(N+1)+m$, running from 1 to $M \equiv N(N+1)$. A Master equation for $\bar{P}_{k}(t)$, the probability to find the system in the state $k$ at time $t$, can be easily written. Being a one-dimensional problem, it can be solved and the steady state distribution found explicitly. Further, generalizing a method used in the theory of random walks on lattice rings [7], [2], (replication) we are 
able to find implicitly time-dependent information in the steady state, namely, asymptotic velocities and diffusion constants associated with both the hole and the defect bond. The final expressions, though explicit functions of the $q$ 's and $W$ 's, are rather complicated, so that it is difficult to gain much insight into their physical content. Thus, we considered a few special cases, for which these explicit formulae display intuitively comprehensible properties of the system, such as the order of magnitude of the effects of the impurity.

Turning our focus to physical realizations of this model, we rely on the existing connection between an impurity-free ASEP and the Duke-Rubinstein reptation model for gel-electrophoresis [5]. There, a polymer is thought of as a chain of segments, confined to a tube of cells. Each cell may contain one or more segments. The links between segments are mapped into particles and/or holes in the ASEP in that a link bridging two cells corresponds to a particle encoded with "charge" \pm 1 , while a link lying within one cell corresponds to a hole. In an external field, the segments move preferentially in the direction of the field, a behavior which translates into the "pure asymmetric case" of the ASEP. Of course, in general, a polymer will have more than one link lying within a cell, motivating the generalization of Derrida's work to a multi-hole ASEP [10]. A more fundamental difference between a periodic ASEP and gel-electrophoresis lies in that open boundary conditions are more appropriate for the latter, leading to many changes in the string of "particles" in the ASEP. Significantly, a recent work [11 proved rigorously that, to leading order in $N$, the diffusion constant of an open chain coincides with that of the periodic chain. Thus, the analysis of a periodic chain [2, 10] should be taken more seriously than an academic exercise. In a previous publication [6], we have shown that a segment of the polymer which is insensitive to the external drive (an impurity) can be mapped into a defect bond in the ASEP. Based on the success of the impurity-free ASEP as a model for gel-electrophoresis, the work here should be further generalized to include multi-hole and multi-impurity cases. The results may be of importance to experiments on the drifting of polymers made from a mixture of two different types of monomers.

Other applications of asymmetric exclusion processes include, e.g., driven diffusive systems with two species [12] and models of traffic flow [13]. The interesting phenomena of phase transitions observed in these systems are, however, present only in two or more dimensions. It clearly behooves us to examine our model in dimensions higher than one. Another obvious extension of our study is the effect of open boundary conditions. The impurity-free ASEP, even in one-dimension, is known to display extremely interesting collective behavior such as spontaneous symmetry breaking [3]. There is little doubt that our study should be regarded as only a small step towards the exploration of the vast unknown of non-equilibrium statistical mechanics.

\section{Acknowdlegements}

Illuminating discussions with M. Evans, C. Hill, C. Laberge, G. Korniss, Y. Shnidman, G. Schütz, T. Tél, and especially B. Schmittmann are gratefully acknowledged. We are indebted to T.Tél and Z.Rácz for the kind hospitality and support at the Eötvös Workshop, Budapest, July 1994, where this work was first presented. One of us (RKPZ) thanks E. 
Domany and D. Mukamel for their hospitality at the Weizmann Institute, Israel. This research is supported in part by the US National Science Foundation through the Division of Material Research and the Hungarian Science Foundation under grant number OTKA F17166.

\section{References}

[1] R. Baxter Exactly Solved Models in Statistical Mechanics, Academic Press, London (1982)

[2] B. Derrida J. Stat. Phys. 31433 (1983)

[3] T.M. Ligett Interacting Particle Systems (Springer, N.Y.) (1985); L.H. Gwa and H. Spohn Phys. Rev. Lett. bf 68, 725 (1992); L.H. Gwa and Spohn H. Phys. Rev. A46, 844 (1992); D. Dhar, Phase Transitions 9, 51 (1987); J. Krug Phys. Rev. Lett. 67, 1882 (1991); Phys. 69, 667 (1992); B. Derrida and M.R. Evans J. Phys. I. France 3, $311,(1993)$

[4] B. Schmittmann and R.K.P. Zia in Phase Transitions and Critical Phenomena, vol. 17: Statistical Mechanics of Driven Diffusive Systems, eds. C.Domb and J.L. Lebowitz, Academic Press, London (1995)

[5] M. Rubinstein, Phys. Rev. Lett. 59, 1946 (1987); T. A. J. Duke, Phys. Rev. Lett. 62, 2877 (1989); B. Widom, J.L. Viovy and A. D. Defontaines, J. Phys. I. France 1, 1759 (1991); G.T. Barkema, J.F. Marko, B. Widom, Phys. Rev. E 49, 5303 (1994); P.G. de Gennes, J. Chem. Phys., 55, 572 (1971).

[6] Z. Toroczkai and R.K.P. Zia, Phys. Lett. A 217, 97, (1996).

[7] B. D. Hughes Random Walks and Random Environments, vol. 1 Random Walks (Clarendon Press, Oxford) (1995).

[8] S.A. Janowsky and J.L. Lebowitz, Phys. Rev. A45, 618 (1992) and J. Stat. Phys. 77, 35 (1994); G. Schütz, J. Stat. Phys. 71, 471 (1993); F.J. Alexander, Z. Cheng, S.A. Janowsky and J.L. Lebowitz, J. Stat. Phys. 68, 761 (1992); D. Kandel and D. Mukamel, Europhys. Lett. 20, 325 (1992)

[9] Y. Shnidman, in Mathematics in Industrial Problems IV , ed. A. Friedman (Springer, Berlin, 1991)

[10] J.M.J. van Leeuwen and A. Kooiman, Physica A184, 79 (1992) and Physica A194, 163 (1993).

[11] M. Prähofer, H. Spohn, (1996) (submitted to Physica A) 
[12] B. Schmittmann, K. Hwang and R.K.P. Zia, Europhys. Lett. 19, 19 (1992); G. Korniss, B. Schmittmann and R. K. P. Zia, Europhys. Lett. 32, 49 (1995); and J. Stat. Phys. (1996) (submitted)

[13] O. Biham, A.A. Middleton, and D. Levine, Phys. Rev. A46 , R6128 (1992) and K.-t. Leung, Phys. Rev. Lett. 73, 2386 (1994); 